\title{
Risco cardiovascular, índices antropométricos e percepção de qualidade de vida em idosos
}

Cardiovascular risk, anthropometric indexes and perception of quality of life in the elderly

\author{
J. O. Couto ${ }^{1}$; W. S. Santos ${ }^{1}$; J. R. Santos ${ }^{3}$; A. E. C. Simões ${ }^{1}$; E. G. M. Santos ${ }^{1}$; R. \\ J. S. Silva ${ }^{1,2^{*}}$ \\ ${ }^{1}$ Departamento de Educação Física/NUPAFISE/Universidade Federal de Sergipe, 49100-000, São Cristivão-Sergipe, \\ Brasil \\ ${ }^{2}$ Programa de Pós-Graduação em Educação Física/ NUPAFISE/Universidade Federal de Sergipe, 49100-000, São \\ Cristivão-Sergipe, Brasil \\ ${ }^{3}$ Departamento de Educação Física/ Faculdade Estácio de Sergipe, 49020-490, Aracaju - Sergipe, Brasil \\ *rjeronimoss@ufs.br
}

(Recebido em 12 de janeito de 2017; aceito em 28 de abril de 2017)

\begin{abstract}
O presente estudo teve como objetivo identificar à prevalência de risco cardiovascular de acordo com índices antropométricos e avaliar à qualidade de vida em idosas participantes de grupos comunitários de prática de atividade física. Trata-se de um estudo com delineamento transversal, com amostra por conveniência. A amostra foi constituída por 84 idosas com média de idade 66,66 \pm 5,54 anos. Aplicou-se instrumento compilado a partir de questionários validados para o Brasil. Também coletou-se os dados de massa corporal, estatura e circunferências de cintura, quadril e abdômen. Foram analisadas as variáveis sociodemográficas, percepção de qualidade de vida, nível de atividade física e a avaliação dos índices antropométricos índice de massa corporal e relação cintura/quadril. Enquanto principais resultados, verifica-se que 64,6\% dos idosos são ativos, à circunferência de cintura classifica 79,48\% dos idosos como "risco" e não houve diferença entre a percepção de qualidade de vida e à exposição ao risco identificado pelos índices antropométricos $(\mathrm{F}(3,71)=$ $0,31 ; \mathrm{p}=0,82)$. Conclui-se que a circunferência de cintura foi o índice antropométrico que melhor estimou o risco do grupo e que a percepção de qualidade de vida independe da estimativa de risco a qual o sujeito está exposto.

Palavras-chave: idoso, obesidade, qualidade de vida
\end{abstract}

The present study aimed to identify the prevalence of cardiovascular risk according to anthropometric indices and to evaluate the quality of life in elderly women participating in community groups of physical activity practice. It is a cross-sectional study, with convenience sample. The sample consisted of 84 elderly women with a mean age of $66.66 \pm 5.54$ years. An instrument compiled from questionnaires validated for Brazil was applied. Data were also collected on body mass, height and circumferences of waist, hip and abdomen. sociodemographic variables, perception of quality of life, level of physical activity and evaluation of anthropometric indices, body mass index and waist / hip ratio were analyzed. The main results show that $64.6 \%$ of the elderly are active, waist circumference classifies $79.48 \%$ of the elderly as "risk" and there was no difference between the perception of quality of life and the risk exposure identified by the anthropometric index $(\mathrm{F}(3,71)=0.31, \mathrm{p}=0.82)$. It was concluded that waist circumference was the anthropometric index that best estimated the risk of the group and that the perception of quality of life independent of the risk estimate to which the subject is exposed.

Keywords: elderly, obesity, quality of life

\section{INTRODUÇÃO}

A transição demográfica (TD), caracterizada pelo aumento da expectativa de vida, redução da taxa de fecundidade e consequente aumento da proporção de idosos em relação aos mais jovens, tem se configurado como um fator preocupante entre os países desenvolvidos e em desenvolvimento [1]. Essa preocupação se dá devido às alterações que ocorrem na previdência social e no alto custo financeiro despendido na saúde especializada, tendo em vista que um idoso requer o triplo de investimentos financeiros e de cuidados [2].

No Brasil a TD tem se configurado como uma ação rápida e em um curto período de tempo, verificando-se um crescimento acima de $4 \%$ ao ano, da população com 60 anos ou mais, atingindo 
19,6 milhões em 2010, verificando-se que esse número chegará a 41,5 milhões em 2030, e 73,5 milhões em 2060 [3]. Em termos mundiais, o segmento populacional acima dos 60 anos era composto por 901 milhões de pessoas representando, em 2015, $12 \%$ da população, no entanto, os indicadores estimam que esse número chegue a 1,4 bilhão em 2030 e 2,1 bilhões em 2050 [4].

Associado ao crescimento da longevidade há o aumento da vulnerabilidade ao surgimento de doenças crônicas não transmissíveis, que podem ser mapeadas pelo uso dos índices antropométricos por conta de sua relação com o excesso de peso e obesidade [5].

A obesidade representa um estado de alto e extenso aumento de gordura corporal total. Sendo considerada como uma epidemia mundial, é caracterizada como uma condição complexa de ordem multifatorial [5]. Condição que leva ao surgimento ou acréscimo de doenças, estando a obesidade abdominal, intimamente relacionada com o aumento significativo de doenças cardiovasculares e de ordem metabólica, como a hipertensão, dislipidemia e hiperglicemia [7,8].

A obesidade pode ser diagnosticada de diversas formas por dupla energia absortometria de raios$x$ de dupla energia (DEXA), tomografia computadorizada, ressonância magnética nuclear e por índices antropométricos: Índice de Massa Corporal (IMC), Circunferência de Cintura (CC) e o índice da Relação Cintura Quadril (RCQ) [9].

Contudo, os índices antropométricos são os mais utilizados na ação clínica por serem objetivos, de baixo custo e de fácil aplicabilidade [9]. No estudo de Sharma et al. [10] através da combinação dos índices antropométricos foi possível identificar um aumento do risco de mortalidade em idosos com doença coronariana que apresentaram obesidade abdominal.

A análise do perfil antropométrico além de estimar a prevalência de doenças cardiovasculares, está associada com o nível de qualidade de vida do idoso. Tendo em vista que a qualidade de vida é a percepção subjetiva de forma negativa ou positiva, que o indivíduo apresenta sobre seus aspectos físico, social e psicológico [11].

De acordo com Giuli et al. [12], idosos que apresentam excesso de peso e obesidade tendem a ter percepção negativa da qualidade de vida.

Estudos destinados à avaliação do estado nutricional e condições de saúde do idoso tornam-se importantes, devido à necessidade de identificar a predisposição ao surgimento de doenças cardiovasculares decorrentes da obesidade no qual irão afetar diretamente a qualidade de vida dos idosos [8].

Partindo do exposto, o presente estudo teve como objetivo identificar a prevalência de risco cardiovascular de acordo com índices antropométricos e avaliar a qualidade de vida em idosas participantes de grupos comunitários de prática de atividade física.

\section{MATERIAL E MÉTODOS}

Trata-se de um estudo com delineamento transversal, com amostra por conveniência. A população foi constituída por participantes de um programa comunitário de Atividade Física em Nossa Senhora do Socorro (SE). Para elegibilidade da amostra, o participante deveria estar presente no dia da coleta, ter mais de 60 anos de idade e não possuir limitação motora. Sendo assim, a amostra foi constituída por 84 idosas com média de idade 66,66 \pm 5,54 anos.

O Programa Comunitário de Atividade Física do Município de Nossa Senhora do Socorro (SE), é executado em 16 polos distribuídos na Zona Rural e Urbana. São realizadas atividades de caminhada orientada e ginastica aeróbica e localizada, sob a supervisão de profissionais de Educação Física, no turno da manhã nos dias letivos da semana.

Inicialmente foi realizado contato com a Secretaria Municipal de Saúde para que fossem apresentados os objetivos e procedimentos da pesquisa. Após a aprovação da pesquisa, houve uma reunião com os coordenadores de cada polo para identificação da quantidade de participantes em cada unidade, estimativa do tempo de permanência das equipes em cada polo e agendamento do dia e horário da coleta de dados.

Foram analisadas as variáveis sociodemográficas, percepção de qualidade de vida, nível de atividade física e índices antropométricos (IA). A aplicação dos questionários ocorreu em forma de entrevista. Anteriormente a coleta de dados os avaliadores passaram por um treinamento para 
avaliar a reprodutibilidade dos instrumentos utilizados, avaliou-se o índice de reprodutibilidade no qual apresentou uma correlação de 0,92 .

Os dados sociodemográficos foram coletados através do Questionário de Classificação Econômica Brasil [13] composto por dois blocos de perguntas, o primeiro confere pontos em função de cada propriedade domiciliar (ex. quantidade de automóvel, de TV, etc.), e o segundo ao grau de instrução do chefe de família. A classificação é feita através da totalização dos pontos obtidos em classes econômicas conforme segue: "A1", “A2", "B1", "B2" (classe econômica alta), "C1", "C2" (classe econômica média), "D" e "E" (classe econômica baixa).

Com a finalidade de avaliar a percepção da qualidade de vida, utilizou-se o Questionário WHOQOL-BREF [14], constituído por 26 questões sobre qualidade de vida. Dentre essas 26 questões, duas avaliam a percepção do indivíduo sobre a sua qualidade de vida e a sua saúde (1 e 2), e as demais vinte e quatro questões ( 3 a 26) contemplam os quatro domínios: físico, psicológico, social (relações sociais), e meio-ambiente. Cujas respostas são pontuadas de acordo com a escala Likert variando de 1 ("pior") até 5 ("melhor"). Ao responder o questionário o indivíduo deve ter como base as últimas duas semanas e deve ter em mente seus valores, aspirações, prazeres e preocupações.

Para avaliar o nível de atividade física foi utilizada a forma curta do Questionário Internacional de Atividade Física (IPAQ) que foi desenvolvido com o objetivo de estimar o nível de prática comum de atividade física de populações de diversos países e ambientes socioculturais [15].

A avaliação dos Índices Antropométricos foi realizada a partir das medidas de massa corporal, estatura e circunferência de cintura, abdômen e quadril utilizando a padronização sugerida pela Sociedade Internacional de Antropometria [16]. Obteve-se a classificação do estado nutricional a partir do Índice de Massa Corporal (IMC). O risco cardiovascular foi estimado a partir dos índices antropométricos para a circunferência de cintura (CC): foi considerado "baixo risco" mulheres com $\mathrm{CC}<80 \mathrm{~cm}$, "risco aumentado" mulheres com CC entre 80 a $88 \mathrm{~cm}$ e "risco substancialmente aumentado" para CC $>88 \mathrm{~cm}$. Para o IMC foi considerado como "baixo risco" os valores $<25$ $\mathrm{kg} / \mathrm{m} 2$ e "risco" e "alto risco" os valores $\geq 25 \mathrm{~kg} / \mathrm{m} 2$. Os valores da relação cintura quadril (RCQ) foram obtidos pela divisão entre as medidas da circunferência de cintura pela circunferência do quadril, tendo como ponto de corte de risco RCQ $\geq 80 \mathrm{~cm}$ para mulheres.

Este estudo foi aprovado pelo Comitê de Ética e Pesquisa da Universidade Federal de Sergipe UFS (parecer CAAE-0213.0.107.000-11), de acordo com a Resolução nº 466/2012 pertencente às pesquisas realizadas com seres humanos e todos os participantes autorizaram a sua participação através da assinatura do termo de consentimento livre e esclarecido.

Quanto aos procedimentos de análise de dados, foram utilizados elementos da estatística descritiva na forma de média, desvio padrão e frequências. Análise de variância para a verificação das possíveis diferenças entre os domínios do WHOQOL-BREF e a classificação da obesidade pelos índices estudados. Em todas as análises foi considerado o nível de significância de 5\% $(\mathrm{p}<0,05)$. Os dados foram analisados a partir do programa estatístico SPSS, versão 20.0.

\section{RESULTADOS}

Características gerais do grupo de idosas participantes do programa Comunitário de Atividade Física da cidade de Nossa Senhora do Socorro (Tabela 1). A amostra avaliada apresentou média de idade 66,66 $\pm 5,54$ anos e mais da metade se autodeclarou de cor parda. Sendo que mais de 50\% do grupo são ativas, de classe média, aposentadas, principal responsável financeiro da casa e apresentam baixos níveis de escolaridade.

$\mathrm{Na}$ tabela 2, verifica-se a prevalência do risco cardiovascular segundo os índices antropométricos, onde se pode observar que a CC foi o índice que apresentou maior prevalência de risco neste grupo, seguida pela medida de IMC e, logo após, a RCQ observada como o índice que obteve menor estimativa da prevalência de risco. 
Tabela 1: Características sociodemográficas em idosas participantes de um programa comunitário de atividade física.

\begin{tabular}{|c|c|c|}
\hline Variáveis & $\mathbf{N}$ & Prevalência $(\%)$ \\
\hline \multicolumn{3}{|l|}{ Ocupação } \\
\hline Aposentado/pensionista & 64 & 77,1 \\
\hline Desempregado & 14 & 16,9 \\
\hline Outros & 5 & 6 \\
\hline \multicolumn{3}{|l|}{ Cor da pele } \\
\hline Negro/preto & 17 & 20,5 \\
\hline Pardo & 53 & 63,9 \\
\hline Outros & 13 & 15,6 \\
\hline \multicolumn{3}{|l|}{ Responsável financeiro } \\
\hline Sim & 58 & 69,9 \\
\hline Não & 24 & 28,9 \\
\hline \multicolumn{3}{|l|}{ Plano privado } \\
\hline Sim & 16 & 19,3 \\
\hline Não & 67 & 80,7 \\
\hline \multicolumn{3}{|l|}{ Grau de Instrução } \\
\hline Analfabeto até a terceira série do Ensino Fundamental & 41 & 49,4 \\
\hline Até a quarta série do Ensino Fundamental & 30 & 36,1 \\
\hline Fundamental completo & 3 & 3,6 \\
\hline Médio completo & 7 & 8,4 \\
\hline \multicolumn{3}{|l|}{ Classe econômica } \\
\hline Alta & 2 & 2,4 \\
\hline Média & 43 & 51,9 \\
\hline Baixa & 36 & 43,4 \\
\hline \multicolumn{3}{|l|}{ Nível de Atividade Física (NAF) } \\
\hline Baixo nível de Atividade Física & 29 & 35,4 \\
\hline Ativo & 53 & 64,6 \\
\hline
\end{tabular}


Tabela 2: Prevalência de risco cardiovascular em idosas segundo os índices antropométricos.

\begin{tabular}{cccc}
\hline $\begin{array}{c}\text { ÍNDICES } \\
\text { ANTROPOMÉTRICOS }\end{array}$ & \multicolumn{2}{c}{ RISCO CARDIOVASCULAR } & $\begin{array}{c}\text { VALORES } \\
\text { DESCRITIVOS }\end{array}$ \\
\hline & SIM & NÃO & $\mathbf{x} \pm \mathbf{S}$ \\
\hline IMC & $\mathbf{n}(\boldsymbol{\%})$ & $19(24,36)$ & $27,75 \pm 4,69$ \\
RCQ & $59(75,64)$ & $25(32,05)$ & $0,86 \pm 0,08$ \\
CC & $53(67,95)$ & $16(20,52)$ & $88,06 \pm 11,35$ \\
\hline
\end{tabular}

a: Índice de Massa Corpórea, b: Relação Cintura/Quadril, c: Circunferência de Cintura, x: Média, s: Desvio Padrão.

Na tabela 3 apresenta os valores descritivos obtidos a partir da classificação de risco pelos índices antropométricos nos participantes do estudo. Esta tabela apresenta os altos valores obtidos por faceta do WHOQOL-BREF, sugerindo que o grupo ter boa percepção de qualidade de vida. No entanto, também é percebido que independente da exposição ao risco identificado na quantidade de índices antropométricos que classifica o sujeito, não há diferença na percepção da qualidade de vida nos domínios sugerindo que apesar da exposição ao risco, não a diferença na percepção desta entre quem está classificado como "risco" por um, dois ou três índices antropométricos e quem não foi classificado como tal.

Tabela 3: Percepção de qualidade de vida (WHOQOL-BREF) conforme classificação de risco verificado pelos índices antropométricos.

\begin{tabular}{ccccccc}
\hline \multirow{2}{*}{ Escores } & \multicolumn{7}{c}{ CLASSIFICAÇÃO DO RISCO } \\
\cline { 2 - 7 } WHOQOL-BREF & SEM RISCO & $\mathbf{1}$ & $\mathbf{2}$ & $\mathbf{3}$ & $\mathbf{F}_{(3,71)}$ & $\boldsymbol{p}$ \\
\hline Físico & $61,56 \pm 15,47$ & $67,09 \pm 16,99$ & $71,78 \pm 14,42$ & $60,71 \pm 12,87$ & 1,53 & 0,21 \\
Psicológico & $67,65 \pm 13,36$ & $71,13 \pm 13,91$ & $68,75 \pm 17,15$ & $70,83 \pm 11,96$ & 0,29 & 0,83 \\
Social & $70,83 \pm 18,05$ & $77,38 \pm 13,64$ & $72,50 \pm 11,81$ & $70,37 \pm 6,05$ & 0,66 & 0,58 \\
Meio Ambiente & $51,56 \pm 16,88$ & $45,98 \pm 19,55$ & $53,43 \pm 15,55$ & $50,69 \pm 12,47$ & 0,49 & 0,69 \\
Total & $62,9 \pm 13,73$ & $65,39 \pm 12,65$ & $66,61 \pm 12,30$ & $63,15 \pm 8,23$ & 0,31 & 0,82 \\
\hline
\end{tabular}

\section{DISCUSSÃO}

Enquanto principais resultados, a CC foi o índice antropométrico que melhor estimou o risco cardiovascular e a percepção da qualidade de vida independe da estimativa do risco ao qual o sujeito está exposto.

Como no estudo realizado por Pereira [17], que analisando indicadores antropométricos associados a fatores de risco cardiovasculares em idosos chegou ao resultado que $30 \%$ dos homens e $44,7 \%$ das mulheres apresentam síndrome metabólica e que a relação cintura/estatura e o índice de massa corporal são os indicadores antropométricos que possuem maior associação com a síndrome metabólica.

Assim também para Jackson [18] que realizou um estudo com 5160 homens e mulheres que teve como objetivo examinar a estabilidade à médio prazo dos parâmetros antropométricos e cardiometabólica na população em geral. Variáveis antropométricas, como IMC e circunferência da cintura foram estabelecidas como indicadores valiosos para o risco de doenças crônicas.

No presente estudo entre os índices antropométricos avaliados a CC foi a que teve a maior representatividade na estimativa do risco cardiovascular, situação semelhante ao 
verificado em Costa et al. [19], sugerindo que a CC é o melhor índice para a classificação de risco.

Seguindo os mesmos resultados o estudo de Ferreira et al. [20] que teve como objetivo investigar a prevalência de fatores de risco cardiovascular em idosos usuários do Sistema Único de Saúde de Goiânia, um dos fatores que obteve umas das maiores prevalências foi a CC com 83,3\% dos 418 participantes do estudo estando classificado como acima do padrão ideal.

Em estudo realizado por Soar [21] que teve como objetivo descrever as prevalências de fatores de risco para doenças cardiovasculares em idosos não institucionalizados, as idosas foram consideradas como grupo vulnerável para fatores de risco cardiovasculares, devido apresentarem número de medidas com valores acima do recomendado, sendo 47,40\% das mulheres e 36,12\% dos homens foram classificados como sobrepeso e 76,16\% das mulheres e $41,06 \%$ dos homens tiveram com a CC acima do recomendado.

Mesmo não sendo a variável que obteve maior representatividade o IMC estimou alto nível de risco entre os participantes da pesquisa com $71,1 \%$ sendo classificado como alto risco. Dados que se assimilam com o estudo realizado por Andrade et al. [22], que teve como objetivo avaliar a prevalência de sobrepeso e de obesidade associados a fatores socioeconômicos, no estudo também foi encontrado um alto nível de idosos que se encontra acima do peso $65,2 \%$ e foram classificados como sobrepeso/obeso. Por isso, outras variáveis devem ser analisadas e consideradas nas estimativas de risco para 0 desenvolvimento de doenças crônicas.

Embora a RCQ tenha sido a variável que obteve a menor prevalência entre os idosos desse estudo com 63,9 \% dos idosos estando classificado como acima do limite de risco ela é uma avaliação de extrema importância como indicador de fator de risco. Estudo [23] realizado na cidade de Maringá que teve como objetivo identificar os índices antropométricos que se associam a doenças cardiovasculares e metabólicas em idosos. Obteve como resultado a presença do diabetes Mellitus associada com a condição inadequada de RCQ.

Com relação aos escores obtidos para os domínios que caracterizam a percepção de qualidade de vida e sua relação com a estimativa identificada através dos índices antropométricos, foi verificado bons escores médios no grupo para a percepção de qualidade de vida, mas não houve diferença estatística entre a classificação de risco pelos índices antropométricos e a percepção de qualidade de vida. Dos quatro domínios, os que apresentaram maiores escores médios foram os escores físico, psicológico e social. Isso indica que os aspectos físicos, psicológicos e sociais foram os que mais contribuíram positivamente para a qualidade de vida dos entrevistados. O domínio que apresentou menor escore médio foi o de meio ambiente.

Em estudo de Ferretti et al. [24] que analisou a qualidade de vida em idosos praticantes e não praticantes de exercício físico regular, foi possível concluir que idosos praticantes de exercício físico regular apresentaram melhores índices de qualidade de vida do que os não praticantes, e que essa prática promove maior preservação das funções físicas, psicológicas e sociais, o que leva a deduzir que o exercício físico é um determinante da melhor qualidade de vida em idosos.

Em estudo realizado por Pegorari et al. [25] que teve como objetivo verificar a associação entre as condições de saúde e qualidade de vida com a prática de atividade física no lazer em idosos rurais, com a participação de 850 idosos, apresentaram um elevado percentual de indivíduos inativos no lazer com maior número de morbidades, indicativo de depressão e percepção negativa de saúde. Enquanto que nos ativos, foram observados maiores escores de qualidade de vida nos domínios físico, psicológico e meio ambiente do WHOQOL-BREF. 
Para o nível de atividade física $64,6 \%$ foram classificados como ativo, corroborando com o estudo de Mazo, Benedetti e Sacomori [26] que teve como objetivo avaliar a associação entre a participação em grupos comunitários e a atividade física entre idosos de Florianópolis. Com uma amostra de 1062 idosos, do qual 293 participavam de grupos comunitários e 769 não, 66,6\% dos participantes de grupos comunitários foram classificados como ativos. Do mesmo modo, estudo [27] que investigou o nível de percepção de saúde, atividade física e qualidade de vida em idosos, com participação de 190 idosos integrantes de um Centro de Atenção a Terceira Idade, 56\% foram classificados como ativos.

Demonstrando dessa forma a importância da participação de grupos comunitários para o aumento do nível de atividade física. A utilização dos métodos de avaliação antropométrica realizado no presente estudo é um método simples, de fácil execução, barato e um preditor do risco cardiovascular.

\section{CONCLUSÃO}

De acordo com os resultados encontrados ficam evidenciadas que a CC foi o índice antropométrico que indicou uma maior prevalência de risco cardiovascular nas idosas, a RCQ foi a que indicou a menor prevalência para o risco cardiovascular.

$\mathrm{Na}$ análise da classificação de risco pela quantidade de índices antropométricos de acordo com as facetas do WHOQOL BREF, pôde-se verificar que a percepção de qualidade de vida independe da classificação do risco cardiovascular estimada pelos índices antropométricos, indicando que o risco estimado pode não estar relacionado à percepção de qualidade de vida entre idosos participantes de programas comunitários de atividade física.

\section{AGRADECIMENTOS}

Agradecemos a Secretaria de Saúde do Município de Nossa Senhora do Socorro e aos Profissionais de Educação Física ligados ao Programa Comunitário de Atividade Física local, pela disponibilidade e apoio na execução desta pesquisa.

\section{REFERÊNCIAS BIBLIOGRÁFICAS}

1. Closs VE, Schwanke CHA. A evolução do índice de envelhecimento no Brasil, nas suas regiões e unidades federativas no período de 1970 a 2010. Rev. Bras. Geriatr. Gerontol. 2012 Jul/Set;15(3):443458, doi: 10.1590/S1809-98232012000300006

2. Souza MCM. O envelhecimento da população brasileira e os desafios para o setor saúde. Cad. Saúde Pública. 2012 Fev;28(2): 208-209.

3. Borges GM, Campos MB, Silva LGC. Transição da estrutura etária no Brasil: oportunidades e desafios para a sociedade nas próximas décadas. In: Ervatti LR, Borges GM, Jardim AP. Mudança Demográfica no Brasil no início do século XXI: Subsídios para as projeções da população. Rio de Janeiro: Instituto Brasileiro de Geografia e Estatística; 2015. p. 138-151.

4. United Nations, Department of Economic and Social Affairs, Population Division. World Population Prospects: The 2015 Revision. 2015.

5. Falsarella, GR, et al. Envelhecimento e os fenótipos da composição corporal. Kairós Gerontologia. Revista da Faculdade de Ciências Humanas e Saúde. 2014 Jun;17(2):57-77.

6. Assunção, WAC, et al. Abdominal fat behavior in women with advancing age. Revista da Educação Física/UEM. 2013 Jun;24(2): 287-294, doi: 10.4025/reveducfis.v24.2.13425

7. Alpert MA, et al. Impact of obesity and weight loss on cardiac performance and morphology in adults. Progress in Cardiovascular Diseases. 2014 Fev;56(4): 391-400, doi: 10.1016/j. p cad.2013.09.003 
8. Scherer F, Vieira JLC. Estado nutricional e sua associação com o risco cardiovascular e síndrome metabólica em idosos. Revista de Nutrição. 2010 Jun;23(3):347-355, doi.org/10.1590/S141552732010000300003

9. Silva IA, Barros DD, Silva VC, Ferreira EAAP. Antropometria na avaliação da obesidade abdominal e risco de doenças. Revista Brasileira de Educação e Saúde. 2014 Marc;4(1), doi: 10.5007/19800037.2011v13n3p238

10. Sharma S, et al. Normal-Weight Central Obesity and Mortality Risk in Older Adults With Coronary Artery Disease. In: Mayo Clinic Proceedings. Elsevier. 2016 Marc;91(3):343-351, doi:10.1016/j.mayocp.2015.12.007

11. Group, TW. The World Health Organization quality of life assessment (WHOQOL): development and general psychometric properties. Social Science \& Medicine. 1998 Jun;46(12), 1569-1585, doi.org/10.1016/S0277-9536(98)00009-4

12. Giuli C, et al. Correlates of perceived health related quality of life in obese, overweight and normal weight older adults: an observational study. BMC Public Health. 2014 Jan;14(1):1, doi: 10.1186 / 1471-245814-35

13. Associação Brasileira de Empresas de Pesquisa (ABEP). Critério de Classificação Econômica Brasil (CCEB). 2011.

14. World Health Organization. WHOQOL: measuring quality of life. Geneva: WHO, 1997.

15. Craig CL, et al. International physical activity questionnaire: 12-country reliability and validity. Medicine e Science in Sports e Execirse. 2003 p. 1381-1395.

16. Marfell JM, et al. International Standards for Anthropometric Assessment. Potchefstroom, South Africa: ISAK. 2006.

17. Pereira MWM. Indicadores antropométricos associados a fatores de risco cardiovasculares. Revista Eletrônica Gestão \& Saúde. 2014 Nov;5(5), 3115-3131.

18. Jackson SE, et al. Four-year stability of anthropometric and cardio-metabolic parameters in a prospective cohort of older adults. Biomark Med. 2015 Fev;9(2):109-22, doi: 10.2217/bmm.14.78

19. Costa CS, Schneider BC, Cesar JA. Obesidade geral e abdominal em idosos do Sul do Brasil: resultados do estudo COMO VAI?. Ciência \& Saúde Coletiva. 2016 Nov;21(11): 3585-3596, doi: 10.1590/1413812320152111.02492016

20. Ferreira CCC, et al. Prevalência de fatores de risco cardiovascular em idosos usuários do Sistema Único de Saúde de Goiânia. Arq. Bras. Cardiol. 2010 Jun;95(5): 621-628

21. Soar C. Prevalência de fatores de risco cardiovascular em idosos não institucionalizados. Rev. bras. geriatr. gerontol. 2015 Mar;18(2):395-395 doi.org/10.1590/1809-9823.2015.14072

22. Andrade BF, et al. Prevalence of overweight and obesity in elderly people from Vitória-ES, Brazil. Ciênc. saúde coletiva. 2012 Mar;17(3) doi.org/10.1590/S1413-81232012000300022

23. Benedetti TRB, Meurer ST, Morini S. Índices antropométricos relacionados a doenças cardiovasculares e metabólicas em idosos. Rev. Educ. Fis/UEM 2012 23(1):123-130, doi: 10.4025/reveducfis.v23i1.11393

24. Ferretti F, et al. Análise de qualidade de vida em idosos praticantes e não praticantes de exercício físico regular. Estud. interdiscipl. envelhec. 2015 20(3):729-743

25. Pegorari MS, et al. Prática de atividade física no lazer entre idosos de área rural: condições de saúde e qualidade de vida. Rev. Educ. Fis/UEM. 2015 26(2):233-241, doi: 10.4025/reveducfis.v26i2.25265

26. Mazo GZ, Benedetti TB, Sacomori C. Association between participation in community groups and being more physically active among older adults from Florianópolis, Brazil. Clinics 2011 66(11):1861-1866, doi:10.1590/S1807-59322011001100003

27. Azevedo GAC, et al. Nível de Percepção de Saúde, Atividade Física e Qualidade de Vida de Idosos. Rev. Bras. de Ciên. da Saú. 2011 15(4):393-398, doi:10.4034/RBCS.2011.15.04.03 